\title{
Les principaux contributeurs
}

Jean COUTURIER est, depuis 2012, attaché à la Direction générale de l'Institut de radioprotection et de sûreté nucléaire, en soutien au déploiement d'un programme de management des connaissances. Il est également expert senior en matière de doctrines de sûreté et d'analyses de risques. Il a commencé sa carrière dans la conception des réacteurs à neutrons rapides, au sein de la société Novatome, à la fois dans les domaines du combustible et de l'analyse de sûreté. Il a intégré l'IPSN en 1986, pour s'impliquer dans l'évaluation de sûreté des réacteurs à neutrons rapides PHENIX et SUPERPHENIX. Ses activités se sont ensuite élargies à la sûreté nucléaire des réacteurs de recherche et de réacteurs à eau sous pression. De 2003 à 2005, il a été Directeur de programme stratégique pour les systèmes dits de génération IV. Il est membre du Groupe permanent d'experts pour les réacteurs nucléaires.

Hassan ABOU YEHIA est, depuis 2017, retraité de l'IRSN. Il a, à partir de 1980, après un doctorat en sciences physiques, occupé divers postes techniques et de management au CEA puis à l'IPSN et à l'IRSN, incluant des évaluations de sûreté pour différentes types d'installations nucléaires en France - ainsi que dans le monde dans le cadre de missions confiées par l'AIEA. De 2006 à 2012, il a été, au sein de cette agence, le responsable de la Section de sûreté des réacteurs de recherche (incluant les installations du cycle de combustible nucléaire). Il a ensuite, au sein de l'IRSN et jusqu'à fin 2016, coordonné notamment les activités de l'IRSN pour les formations en sûreté nucléaire et a participé dans ces formations organisées par l'European Nuclear Safety Training \& Tutoring Institute (ENSTTI).

Emmanuel GROLLEAU est, depuis mi-2018, adjoint du chef de Service du confinement et de l'aérodispersion de polluants de l'IRSN. Après plusieurs années passées au sein du service de calculs et d'études en criticité de la société SGN du groupe AREVA, il a intégré l'IRSN en 2004 dans le Service d'évaluation en charge notamment de la sûreté des réacteurs de recherche. Il a notamment assuré le pilotage de plusieurs évaluations ayant 
donné lieu à des présentations devant le Groupe permanent d'experts en charge des réacteurs. De 2007 à 2011, il a été attaché à la Direction de la stratégie et des partenariats de l'IRSN. De 2012 à mi-2018, il a été adjoint au chef du Service chargé de l'évaluation de la sûreté des installations liées à la recherche et des réacteurs en démantèlement au sein de la Direction de l'expertise de sûreté de l'IRSN. 